\title{
Price-Based Spectrum Management in Cognitive Radio Networks
}

\author{
Fan Wang†, Marwan Krunz†, and Shuguang Cuił \\ $\dagger$ Department of Electrical and Computer Engineering \\ University of Arizona, Tucson, AZ, 85721 \\ \{wangfan,krunz\}@ece.arizona.edu \\ $\ddagger$ Department of Electrical and Computer Engineering \\ Texas A \& M University, College Station, TX, 77843 \\ cui@ece.tamu.edu
}

\begin{abstract}
Cognitive radios (CRs) have a great potential to improve spectrum utilization by enabling users to access the spectrum dynamically without disturbing licensed primary radios (PRs). A key challenge in operating these radios as a network is how to implement an efficient medium access control (MAC) mechanism that can adaptively and efficiently allocate transmission powers and spectrum among CRs according to the surrounding environment. Most existing works address this issue via sub-optimal heuristic approaches or centralized solutions. In this paper, we propose a novel joint power/channel allocation scheme that improves the performance through a distributed pricing approach. In this scheme, the spectrum allocation problem is modeled as a non-cooperative game, with each $C R$ pair acting as a player. A price-based iterative water-filling (PIWF) algorithm is proposed, which enables CR users to reach a good Nash equilibrium (NE). This PIWF algorithm can be implemented distributively with CRs repeatedly negotiating their best transmission powers and spectrum. Simulation results show that the social optimality of the NE solution is dramatically improved through pricing. Depending on the different orders according to which CRs take actions, we study sequential and parallel versions of the PIWF algorithm. We show that the parallel version converges faster than the sequential version. We then propose a corresponding MAC protocol to implement our resource management schemes. The proposed MAC allows multiple CR pairs to be first involved in an admission phase, then iteratively negotiate their transmission powers and spectrum via control-packet exchanges. Following the negotiation phase, CRs proceed concurrently with their data transmissions. Simulations are used to study the performance of our protocol and demonstrate its effectiveness in terms of improving the overall network throughput and reducing the average power consumption.
\end{abstract}

\section{INTRODUCTION}

The concept of a cognitive radio (CR) has recently triggered great interest within the research community (see [10] for a comprehensive survey). The term "cognitive radio" was first coined by Mitola [16] as "the point in which wireless personal digital assistants (PDAs) and the related networks are sufficiently computationally intelligent about radio resources and related computer-to-computer communications to: (a) detect user communications needs as a function of use context, and (b) to provide radio resources and wireless services most appropriate to those needs." Mitola's definition, however, does not specify the radio architecture for the physical and link layers. More recently, the FCC [8] suggested that any radio with adaptive spectrum awareness is to be referred to as a CR. Specifically, a CR should be able to adapt its transmission parameters to the neighborhood environment. CRs are expected to be deployed in both military and commercial applications.

Several scenarios can be found for operating a cognitive radio network (CRN). In this paper, we focus on an opportunistic CRN where the CRs are secondary users that coexist with primary radios (PRs). The PRs are licensed to operate over certain frequency bands. They do not cooperate with or even provide feedback to the CRs. CRs continuously sense the channel and exploit spectrum "holes" for their transmissions. One of the main challenges in an opportunistic CRN is how to design an efficient and adaptive channel access scheme that supports dynamic channel selection and power/rate allocation in a distributed (ad hoc) CRN environment. An efficient design is one that tries to maximize the CRN's performance without disturbing PR transmissions. A typical measure of efficiency is the achievable sum-rate across all CR pairs. Unfortunately, the problem of maximizing the sum-rate over a multi-user, interference channel subject to individual power constraints is a non-convex optimization problem [29]. Such a problem becomes even more intractable when we allow multiple CRs to share the same channel, as we now have to consider CRto-CR interferences in addition to PR-to-CR and CR-to-PR interferences.

Several attempts have been made to solve the aforementioned interference channel problem. One well-known resource allocation scheme, called iterative water-filling (IWF), was first proposed in [31], where a non-cooperative game was used to model the spectrum management problem with each user iteratively maximizing its own rate. This per-user optimization problem is convex and leads to a water-filling solution. For the two-user case, it was proven that the Nash Equilibrium (NE) exists and the IWF algorithm converges to the NE under certain conditions. However, this NE is generally not Pareto optimal [20] and may be quite inefficient in term of sum-rate [4]. This is because in a non-cooperative game, each user only has the incentive to maximize its own utility function without considering the overall system performance. A centralized spectrum management scheme was proposed in [4], which 
greatly improves the system performance over the IWF scheme by utilizing a centralized spectrum management center (SMC). However, such an approach cannot be implemented in an ad hoc opportunistic CRN, where none of the CRs has global knowledge of the entire CRN to function as the SMC.

Given the above, we are motivated to design a channel/power/rate allocation scheme that overcomes the inefficiency of IWF and yet can be implemented in a distributed fashion. Specifically, we provide incentives to CR users such that they can reach a more socially efficient NE. A commonly used incentive technique in game theory is pricing (a thorough review is provided in [11]). Previously, pricing techniques have been implemented in various wireless networks such as cellular networks, ad hoc networks, and peer-to-peer networks (e.g., [23], [5], [30], and [1]). In this paper, we apply pricing techniques to CRNs. We propose a price-based iterative water-filling (PIWF) algorithm, and show that this algorithm maintains the simplicity and distributed operation of the IWF algorithm while achieving better bandwidth efficiency (i.e., higher sum-rate). The effectiveness of the pricing technique depends on the selection of the "pricing functions," which is a challenging problem by itself. Although there may exist an "optimal" pricing function that allows the NE to converge to a Pareto-optimum solution, the search for such a pricing function generally requires a central controller and is hard to be implemented in a distributed manner. Some sub-optimal pricing functions have been proposed in the literature. For example, the authors in [5] proposed an auction-like pricing scheme for mobile ad hoc networks (MANETs). The unit price in this scheme (uniform across all users) is gradually increased until the system settles down at a feasible NE. A similar approach was taken in [23], where the users of a wireless data network keep increasing their prices in a uniform fashion until one user begins to receive a decreasing utility. Both of the previously mentioned pricing schemes achieve feasible NEs and improve the system performance. However, the achieved NEs are not guaranteed to be globally optimal, which is partially caused by the fact that both of the two approaches take a uniform unit price for all players in the game. In our work, we determine a user-dependent pricing function, which not only improves the NE, but also achieves globally or locally optimal NE after a few iterations. Such a pricing function can be determined by allowing each CR user to distributively acquire its neighborhood information via control-packet exchanges.

Another problem of applying the IWF algorithm in [31] to CRNs is that this algorithm only considers a total power constraint for each user. In a CRN, PRs impose strict power constraints over each frequency band, so CR users have to abide by frequency-dependent power constraints. Such constraints will affect the response of each CR user and thus the achieved NE. In this paper, we incorporate a frequency-dependent power mask constraint into the optimization problem.

In our proposed algorithm, each user maximizes its own utility function (which includes a pricing function) by performing a single-user price-based water-filling, while treating the interference from other CR users at each sub-band as additive white Gaussian noise (AWGN). The same procedure iterates sequentially, eventually converging to the NE. If the number of users in the network is large, sequential updating may suffer from slow convergence. Therefore, we also discuss a parallel PIWF algorithm (the parallel concept was introduced in [25]), which is an instance of the Jacobi scheme: At each iteration, CRs update their strategies simultaneously, based on the interference measured in the previous iteration. Simulations indicate that this parallel version converges faster than the sequential PIWF algorithm. Both the sequential and parallel PIWF algorithms require CRs to be synchronized and the system parameters to be correctly estimated for each CR. These conditions may not be satisfied in practical systems. To overcome this problem, a "relaxed" update scheme can be used (as in [2], [15], and [24]) and is studied in our work. For the "relaxed" version of the PIWF algorithm, each CR is required to remember its most recent policy choices together with the choices of other users. The relaxed algorithm is more robust to inaccurate estimates and channel oscillations, but it may impact the convergence speed.

Our PIWF algorithms are then integrated into the design of a distributed medium access (MAC) protocol for CRNs. This protocol allows CRs to dynamically select channels and adapt to different transmission powers and rates. We discuss how the various versions of PIWF impact the MAC design. Simulations are conducted to compare the performance of the proposed protocol against other adaptive protocols.

The rest of this paper is organized as follows. The system model is described in Section II. Section III formulates the non-cooperative game and introduces the pricing techniques. We then discuss the PIWF algorithms for solving the NE in Section IV and design the corresponding MAC protocol in Section V. In Section VI, we provide simulation results of the PIWF algorithms and compare them with the classic IWF algorithm. Finally, we draw conclusions and discuss future extensions in Section VII.

\section{SYSTEM MODEL}

We consider a hybrid network consisting of several PRNs and one CRN. The $\mathrm{CRN}$ contains $N \mathrm{CR}$ pairs. The total spectrum consists of $K$ orthogonal frequency channels with central frequencies $f_{1}, f_{2}, \ldots, f_{K}$, where $K<N$. Each PR in a PRN may operate over one or multiple channels. Let $\Omega_{N}=\{1,2, \ldots, N\}$ and $\Omega_{K}=\{1,2, \ldots, K\}$ denote the sets of CR links and channels, respectively.

Each CR may simultaneously transmit over multiple channels. It can also receive over multiple channels (from the same transmitter) at the same time. However, we require that each CR operates in a half-duplex manner, meaning that it cannot receive while transmitting, and vice versa. Let $M_{i}\left(f_{k}\right)$ denote the total noise-plus-interference level measured by CR user $i$ over channel $k$. This quantity includes the PR-to-CR interference, the CR-to-CR interference, and the thermal noise. We assume that when not transmitting, CR $i$ is capable of measuring $M_{i}\left(f_{k}\right)$ over all channels $k \in \Omega_{K}$. Let $\mathbf{M}_{i} \stackrel{\text { def }}{=}$ $\left[M_{i}\left(f_{1}\right), M_{i}\left(f_{2}\right), \ldots, M_{i}\left(f_{K}\right)\right]$, which is used by $\mathrm{CR} i$ to perform channel selection, power control, and rate allocation, as described later. 
The motivation of using $\mathrm{CR}$ technology is to enhance the spectrum utilization by allowing CR users to share the spectrum with PRs. Some previous work [28] assumed that CR transmissions do not interfere with each other, i.e., only one CR user can operate over a given channel in a given neighborhood (along with the PRs). In this way, there is no spectrum sharing among CR users. Such schemes limit the number of admitted CR links, especially when the number of channels is small. In our work, we allow multiple CR users to share a particular channel. Figure 1 depicts a channel allocation example for a CRN with $K=3$ and $N=4$. The dark square indicates that a channel is utilized by a CR. For example, link 1 uses channels 1 and 2, while link 4 uses channel 1 only. We denote the set of utilized channels for CR link $i$ as $S_{i}$. In the above example, $S_{1}=\{1,2\}$ and $S_{4}=\{1\}$. The transmission power vector of CR link $i$ over all channels is denoted by $\mathbf{P}_{i}=\left[P_{i}\left(f_{1}\right), P_{i}\left(f_{2}\right), \ldots, P_{i}\left(f_{K}\right)\right]$, where $P_{i}\left(f_{k}\right)$ is the transmission power of CR $i$ on channel $k$. If channel $k$ belongs to $S_{i}, P_{i}\left(f_{k}\right)>0$; otherwise, $P_{i}\left(f_{k}\right)=0$.

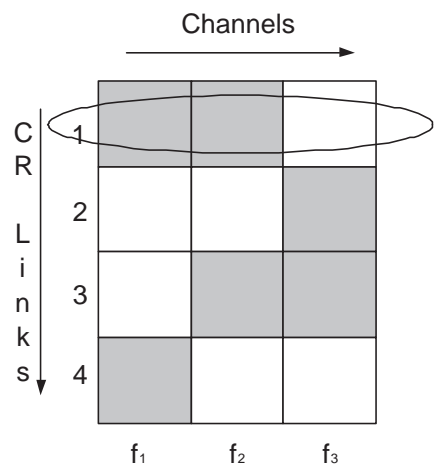

Fig. 1: Example of channel allocation for $4 \mathrm{CR}$ links.

To ensure feasible spectrum sharing, we impose the following constraints:

1) Maximum transmission power constraint: The total transmission power of a CR over the selected channels should not exceed $P_{\max }$, i.e, $\sum_{k \in S_{i}} P_{i}\left(f_{k}\right) \leq P_{\max }$. Here, we assume that the total power constraint is the same for all users. It is easy to extend the treatment to the case where $P_{\max }$ is user-dependent.

2) CR-to-PR power mask constraint: The transmission power of CR $i$ on channel $k$ is constrained by $P_{i}\left(f_{k}\right) \leq$ $P_{\text {mask }}\left(f_{k}\right)$, where $P_{\text {mask }}\left(f_{k}\right)$ is the power mask on channel $k$. Such a per-device power mask is easier to verify at the design stage from a practical point of view. For example, the power mask is often specified by FCC regulations. In this case, CR vendors need to design the radio while ensuring its RF transmission power meets the FCC power mask. Such a philosophy is often used in various wireless technologies (e.g., UWB, Wi-Fi, Walkie-Talkies, etc.). Note that because the number of active CR links that share a given frequency band varies in time and space, it is impractical to design the hardware to account for a "neighborhooddependent" power mask. We use the vector $\mathbf{P}_{\text {mask }} \stackrel{\text { def }}{=}$
$\left[P_{\text {mask }}\left(f_{1}\right), P_{\text {mask }}\left(f_{2}\right), \ldots, P_{\text {mask }}\left(f_{K}\right)\right]$ to denote the power mask on all channels. In the following analysis, we assume that $\mathbf{P}_{\text {mask }}$ is given a priori.

3) Minimum signal-to-interference-and-noise ratio (SINR) constraint: If the received SINR over a given channel is below the SINR threshold $\left(\mathrm{SINR}_{t h}\right)$, the CR will not use that channel.

We assume that the CRs are either static or are moving slowly compared to the convergence time of the resource assignment algorithms. This assumption is generally acceptable because our iterative algorithms operate on the time scale of few milliseconds, whereas pedestrian and vehicular mobility impacts the network topology on the time scale of seconds. In addition, CRs are homogeneous, meaning that they follow the same operation rules and have the same system constraints.

\section{Problem Formulation}

In a "non-cooperative" CRN, each CR user is interested in maximizing its own achievable rate. Such a greedy behavior can be modeled using game theory. Game theory analyzes the interactions of players in decision-making processes. It can be used to identify distributed optimal strategies for the players [18]. A normal game $\mathcal{G}$ is expressed as: $\mathcal{G}=\left\{\Omega, \mathcal{P},\left\{U_{i}\right\}\right\}$, where $\Omega=\{1,2, \ldots, N\}$ is a finite set of rational players; $\mathcal{P}=\mathcal{P}_{1} \times \mathcal{P}_{2} \times \ldots \times \mathcal{P}_{N}$ is the action space with $\mathcal{P}_{i}$ being the action set for player $i$; and $U_{i}: \mathcal{P} \rightarrow \mathcal{R}$ is the utility (payoff) function of player $i$, which depends on the strategies of all players. We can model the channel/power allocation problem in a CRN as a non-cooperative game, in which the players are the $\mathrm{CR}$ users; their actions are the transmission power vector (i.e., the action for user $i$ is given by $\mathbf{P}_{i}=$ $\left.\left[P_{i}\left(f_{1}\right), P_{i}\left(f_{2}\right), \ldots, P_{i}\left(f_{K}\right)\right]\right)$; and their utility functions are associated with their actions and the quality of the channels. Note that a CR user in the game denotes a CR link consisting of a pair of CRs.

\section{A. Utility Function}

In our game, the utility function of user $i$ can be considered as the reward received by this user from the network. This reward should depend on the user's action $\mathbf{P}_{i}$ and the union set of all other users' actions $\mathbf{P}_{-i}$, where $\mathbf{P}_{-i} \stackrel{\text { def }}{=}$ $\left[\mathbf{P}_{1}, \ldots, \mathbf{P}_{i-1}, \mathbf{P}_{i+1}, \ldots, \mathbf{P}_{N}\right]^{T}$. While the selection of the utility function is not unique, the selected utility function must have physical meaning for the particular application. A natural selection of the utility function for CR link $i$ (also used in [6], [24], [31]) is its transmission rate, given by:

$$
\begin{gathered}
U_{i}\left(\mathbf{P}_{i}, \mathbf{P}_{-i}\right)=\sum_{k \in \Omega_{K}} u_{i}\left(P_{i}\left(f_{k}\right)\right)= \\
\sum_{k \in \Omega_{K}}\left[\log _{2}\left(1+\frac{h_{i i}\left(f_{k}\right) P_{i}\left(f_{k}\right)}{\sum_{j \in \Omega_{N}, j \neq i} h_{j i}\left(f_{k}\right) P_{j}\left(f_{k}\right)+M_{i}^{(P R)}\left(f_{k}\right)+N_{i}\left(f_{k}\right)}\right)\right]
\end{gathered}
$$

where $h_{j i}\left(f_{k}\right)$ denotes the channel gain between the transmitter of link $j$ and the receiver of link $i$ over channel $k$, $M_{i}^{(P R)}\left(f_{k}\right)$ denotes the PR-to-CR interference at the receiver of CR link $i$ over channel $k$, and $N_{i}\left(f_{k}\right)$ denotes the received 
thermal noise power on channel $k$. In the paper, this relationship is taken as Shannon's capacity formula. In a practical multi-rate wireless system, the power-rate relationship takes the form of a staircase, and the user sets the transmission rate to the maximum possible rate (among a finite set of rates) that satisfies the SNR threshold at the given transmission power value. It is straightforward to extend our design to accommodate such a power-rate relationship.

Given the utility function in (1), users select their transmission powers to maximize their own utility functions, and under certain conditions, they eventually reach at a NE after several iterations. As discussed in Section I, because of the non-cooperative nature of the game, each $\mathrm{CR}$ user behaves selfishly. Thus, the resulting NE may be far from the Pareto optimum [20]. In practice, we are interested in maximizing a weighted sum of the utilities of all users, defined as:

$$
\max _{\mathbf{P}} \sum_{i \in \Omega_{N}} w_{i} U_{i}\left(\mathbf{P}_{i}, \mathbf{P}_{-i}\right)=\max _{\mathbf{P}} \sum_{i \in \Omega_{N}} w_{i} \sum_{k \in \Omega_{K}} u_{i}\left(P_{i}\left(f_{k}\right)\right)
$$

where $w_{i}$ denotes the weight assigned to $\mathrm{CR}$ user $i$, which may be interpreted in different ways (e.g., priority factor of user $i$ ). Note that the power assignment that solves (2) is a Pareto-optimum solution.

To drive the NE towards the Pareto optimum boundary, we use pricing as an incentive for each selfish CR user to work in a cooperative manner. A new utility function with pricing is then defined as follows:

$$
\tilde{U}_{i}\left(\mathbf{P}_{i}, \mathbf{P}_{-i}\right)=\sum_{k \in \Omega_{K}} \tilde{u}_{i}\left(P_{i}\left(f_{k}\right)\right)
$$

with

$$
\begin{aligned}
& \tilde{u}_{i}\left(P_{i}\left(f_{k}\right)\right) \stackrel{\text { def }}{=}-c_{i}\left(f_{k}\right)+ \\
& \log _{2}\left(1+\frac{h_{i i}\left(f_{k}\right) P_{i}\left(f_{k}\right)}{\left(\sum_{j \in \Omega_{N}, j \neq i} h_{j i}\left(f_{k}\right) P_{j}\left(f_{k}\right)\right)+M_{i}^{(P R)}\left(f_{k}\right)+N_{i}\left(f_{k}\right)}\right)
\end{aligned}
$$

where $c_{i}\left(f_{k}\right)$ represents the pricing function for user $i$ on channel $k$. As discussed in Section I, our goal is to choose a user-dependent pricing function that can drive the CR users to converge to an efficient NE. How to define this pricing function will be discussed in Section III-C.

\section{B. Game Formulation}

Given the price-based utility function in (4), each CR user $i$ iteratively selects its power vector $\mathbf{P}_{i}$ to maximize $\tilde{U}_{i}\left(\mathbf{P}_{i}, \mathbf{P}_{-i}\right)$ subject to the constraints listed in Section II. This results in the following non-cooperative game $\mathcal{G}$ :

$$
\begin{array}{ll}
\max _{\mathbf{P}_{i}} & \tilde{U}_{i}\left(\mathbf{P}_{i}, \mathbf{P}_{-i}\right), \quad \forall i \in \Omega_{N} \\
\text { s.t. } & \\
\text { C1: } & P_{i}\left(f_{k}\right) \geq 0, \quad \forall i \in \Omega_{N} \text { and } k \in \Omega_{K} \\
\text { C2: } & \sum_{k \in \Omega_{K}} P_{i}\left(f_{k}\right) \leq P_{\text {max }}, \quad \forall i \in \Omega_{N} \\
\text { C3: } & P_{i}\left(f_{k}\right) \leq P_{\text {mask }}\left(f_{k}\right), \quad \forall i \in \Omega_{N} \text { and } k \in \Omega_{K}
\end{array}
$$

If there is a solution to the above game, then it would be the one that achieves the NE. Note that the above game differs from the game studied in [31] in the form of the utility function and the additional power mask constraint. Thus, the existence proofs in [6] and [31] cannot be directly applied.

The following proposition show that a NE solution always exists for the above game.

Proposition 1: For any given $P_{\max }$ and $\mathbf{P}_{\text {mask }}$ values, there is at least one NE for the game $\mathcal{G}$ in (5).

Proof: The game in our setup can be shown to be a concave game if the following two properties are satisfied:

1) The action space $\mathcal{P}$ is a closed and bounded convex set;

2) The utility function $\tilde{U}_{i}\left(\mathbf{P}_{i}, \mathbf{P}_{-i}\right)$ is concave over its strategy set.

It is straightforward to show that the two properties are satisfied by the game $\mathcal{G}$. Because a concave game always admits at least one NE [22], the proposition follows immediately.

Given the existence of a NE solution, we need to design an algorithm for CR users to reach the NE. However, before we do that, we first investigate the form of the optimal pricing function.

\section{Optimal Pricing Function}

Pricing is an idea that originated from economics (e.g., [11]). It denotes the cost of commodities for individual decision makers. In the power control context (e.g., [23] and [30]), pricing is used as an incentive mechanism to improve the efficiency of the NE. To illustrate, in Figure 2, we depict an example of the Pareto-optimal frontier and the NE for a two-user game. In general, the NE is not Pareto optimal. Previous pricing techniques usually improve the achieved NE (i.e., moving it closer to the Pareto-optimal frontier) using heuristic pricing functions, since an optimal pricing function generally requires global information and could hardly be ) deployed in a distributed manner. For example, in [23] and [30], the pricing function is a suboptimal linear function with a fixed linear pricing factor for all players.

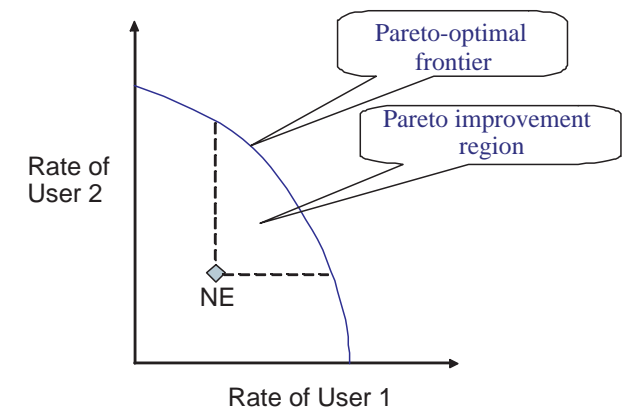

Fig. 2: Nash equilibrium and Pareto-optimal Frontier.

The pricing function can take various forms. A linear pricing function is commonly used because of its implementation simplicity. One contribution of our work is the proposition of a user-dependent linear pricing function that drives the NE close to the Pareto optimal frontier with each player having only its local and certain neighborhood information. The neighborhood information is acquired via control packets that are exchanged during the channel access process (see Section V for details). 
Proposition 2: If there exists a NE for the game $\mathcal{G}$ and if this NE is Pareto optimal, then the linear pricing function factor for user $i$ should be:

$$
\lambda_{i}\left(f_{k}\right)^{o p t}=\frac{1}{w_{i}} \sum_{j \in \mathrm{NBR}_{i}} w_{j} \frac{h_{j j}\left(f_{k}\right) P_{j}\left(f_{k}\right) h_{i j}\left(f_{k}\right)}{M_{j}\left(f_{k}\right)\left(M_{j}\left(f_{k}\right)+h_{j j}\left(f_{k}\right) P_{j}\left(f_{k}\right)\right)}
$$

where $\mathrm{NBR}_{i}$ denotes the set of neighbors for user $i$.

Proof: By definition, a NE is the solution to the individual utility optimization problem for each user given all other users' actions. In our formulation, each individual optimization problem is a convex problem with the linear constraints C1-C3 in (5). So the Lagrangian function for user $i$ can be written as:

$$
\begin{aligned}
& J_{i}=w_{i} \sum_{k \in \Omega_{K}} \tilde{u}_{i}\left(P_{i}\left(f_{k}\right)\right)+\sum_{k \in \Omega_{K}} \alpha_{i, k} P_{i}\left(f_{k}\right) \\
& -\beta_{i}\left(\sum_{k \in \Omega_{K}} P_{i}\left(f_{k}\right)-P_{\text {max }}\right)-\sum_{k \in \Omega_{K}} \gamma_{i, k}\left(P_{i}\left(f_{k}\right)-P_{\text {mask }}\left(f_{k}\right)\right) \\
& \quad=w_{i} \sum_{k \in \Omega_{K}}\left[u_{i}\left(P_{i}\left(f_{k}\right)\right)-\lambda_{i}\left(f_{k}\right) P_{i}\left(f_{k}\right)\right]+\sum_{k \in \Omega_{K}} \alpha_{i, k} P_{i}\left(f_{k}\right) \\
& -\beta_{i}\left(\sum_{k \in \Omega_{K}} P_{i}\left(f_{k}\right)-P_{\text {max }}\right)-\sum_{k \in \Omega_{K}} \gamma_{i, k}\left(P_{i}\left(f_{k}\right)-P_{\text {mask }}\left(f_{k}\right)\right)(7)
\end{aligned}
$$

where $\alpha_{i, k}, \beta_{i}$, and $\gamma_{i, k}$ are the Lagrangian multipliers (nonnegative real numbers). The K.K.T. conditions [3] are given by:

$$
\begin{aligned}
& \frac{\partial J_{i}}{\partial P_{i}\left(f_{k}\right)}=w_{i} \frac{\partial u_{i}\left(P_{i}\left(f_{k}\right)\right)}{\partial P_{i}\left(f_{k}\right)}-w_{i} \lambda_{i}\left(f_{k}\right) \\
&+\alpha_{i, k}-\beta_{i}-\gamma_{i, k}=0, \forall k \in \Omega_{K} \\
& p_{i}\left(f_{k}\right) \geq 0, \forall k \in \Omega_{K} \\
& \alpha_{i, k} p_{i}\left(f_{k}\right)=0, \forall k \in \Omega_{K} \\
& \sum_{k \in \Omega_{K}} P_{i}\left(f_{k}\right)-P_{\max } \leq 0 \\
& \beta_{i}\left(\sum_{k \in \Omega_{K}} P_{i}\left(f_{k}\right)-P_{\max }\right)=0 \\
& P_{i}\left(f_{k}\right)-P_{\text {mask }}\left(f_{k}\right) \leq 0, \forall k \in \Omega_{K} \\
& \gamma_{i, k}\left(P_{i}\left(f_{k}\right)-P_{\text {mask }}\left(f_{k}\right)\right)=0, \forall k \in \Omega_{K}
\end{aligned}
$$

In contrast, to solve the social optimization problem (2) with constraints $\mathrm{C} 1-\mathrm{C} 3$, the Lagrangian function can be written as:

$$
\begin{aligned}
J= & \sum_{i \in \Omega_{N}} w_{i} \sum_{k \in \Omega_{K}} u_{i}\left(P_{i}\left(f_{k}\right)\right)+\sum_{i \in \Omega_{N}} \sum_{k \in \Omega_{K}} \alpha_{i, k} P_{i}\left(f_{k}\right) \\
& -\sum_{i \in \Omega_{N}} \beta_{i} \sum_{k \in \Omega_{K}}\left(P_{i}\left(f_{k}\right)-P_{\text {max }}\right) \\
& -\sum_{i \in \Omega_{N}} \sum_{k \in \Omega_{K}} \gamma_{i, k}\left(P_{i}\left(f_{k}\right)-P_{\text {mask }}\left(f_{k}\right)\right) \\
= & w_{i} \sum_{k \in \Omega_{K}} u_{i}\left(P_{i}\left(f_{k}\right)\right)+\sum_{j \in \Omega_{N}, j \neq i} w_{j} \sum_{k \in \Omega_{K}} u_{j}\left(P_{j}\left(f_{k}\right)\right) \\
& +\sum_{i \in \Omega_{N}} \sum_{k \in \Omega_{K}} \alpha_{i, k} P_{i}\left(f_{k}\right)-\beta_{i} \sum_{k \in \Omega_{K}}\left(P_{i}\left(f_{k}\right)-P_{\text {max }}\right) \\
& -\sum_{i \in \Omega_{N}} \sum_{k \in \Omega_{K}} \gamma_{i, k}\left(P_{i}\left(f_{k}\right)-P_{\text {mask }}\left(f_{k}\right)\right)
\end{aligned}
$$

The K.K.T. conditions for the optimization problem in (2) are given by:

$$
\begin{aligned}
& \frac{\partial J}{\partial P_{i}\left(f_{k}\right)}=w_{i} \frac{\partial u_{i}\left(P_{i}\left(f_{k}\right)\right)}{\partial P_{i}\left(f_{k}\right)}+\sum_{j \in \Omega_{N}, j \neq i} w_{j} \frac{\partial u_{j}\left(P_{j}\left(f_{k}\right)\right)}{P_{i}\left(f_{k}\right)} \\
&+\alpha_{i, k}-\beta_{i}-\gamma_{i, k}=0, \forall i \in \Omega_{N} \text { and } k \in \Omega_{K} \\
& p_{i}\left(f_{k}\right) \geq 0, \forall i \in \Omega_{N} \text { and } k \in \Omega_{K} \\
& \alpha_{i, k} p_{i}\left(f_{k}\right)=0, \forall i \in \Omega_{N} \text { and } k \in \Omega_{K} \\
& \sum_{k \in \Omega_{K}} P_{i}\left(f_{k}\right)-P_{\text {max }} \leq 0, \forall i \in \Omega_{N} \\
& \beta_{i}\left(\sum_{k \in \Omega_{K}} P_{i}\left(f_{k}\right)-P_{\text {max }}\right)=0, \forall i \in \Omega_{N} \\
& P_{i}\left(f_{k}\right)-P_{\text {mask }}\left(f_{k}\right) \leq 0, \forall i \in \Omega_{N} \text { and } k \in \Omega_{K} \\
& \gamma_{i, k}\left(P_{i}\left(f_{k}\right)-P_{\text {mask }}\left(f_{k}\right)\right)=0, \forall i \in \Omega_{N} \text { and } k \in \Omega_{K}
\end{aligned}
$$

By comparing K.K.T. conditions in (8) and (10), to obtain the same solution, we must have:

$$
\lambda_{i}\left(f_{k}\right)=-\frac{1}{w_{i}} \sum_{j \in \Omega_{N}, j \neq i} w_{j} \frac{\partial u_{j}\left(P_{j}\left(f_{k}\right)\right)}{\partial P_{i}\left(f_{k}\right)}
$$

By substituting $u_{j}\left(P_{j}\left(f_{k}\right)\right)$ into (11), we have:

$\lambda_{i}\left(f_{k}\right)=\frac{1}{w_{i}} \sum_{j \in \Omega_{N}, j \neq i} w_{j} \frac{h_{j j}\left(f_{k}\right) P_{j}\left(f_{k}\right) h_{i j}\left(f_{k}\right)}{M_{j}\left(f_{k}\right)\left(M_{j}\left(f_{k}\right)+h_{j j}\left(f_{k}\right) P_{j}\left(f_{k}\right)\right)}$.

If the transmitter of link $i$ and the receiver of link $j$ are not neighbors, i.e., the transmission of link $i$ at the maximum power cannot reach the receiver of link $j$, the channel gain $h_{i j}\left(f_{k}\right)$ is set to zero. Thus, the optimal pricing factor for link $i$ only depends on its neighborhood information. We then have the result in Proposition 2.

Intuitively, a higher pricing factor $\lambda_{i}\left(f_{k}\right)$ will prevent user $i$ from using a large transmission power on channel $k$. In view of (6), for link $i$ to determine its optimal pricing factor, the following procedure is needed: If a neighbor $j$ is to transmit over channel $k$, it needs to broadcast its transmission power $P_{j}\left(f_{k}\right)$, the measured total noise and interference $M_{j}\left(f_{k}\right)$, and the channel gain $h_{j j}\left(f_{k}\right)$ between the transmitter and the receiver of link $j$. The above information can be incorporated into the control packets of the MAC protocol (details in Section V). In addition, $h_{i j}\left(f_{k}\right)$ can be measured from the received signal power of the control packet.

\section{ITERATIVE AlgORITHMS}

From the propositions in the previous section, we can use the following iterative procedure to reach the NE. Each individual CR user, say $i$, first adjusts its linear pricing factor $\lambda_{i}\left(f_{k}\right)$ over all channels according to (6), and then determines its best action [20], i.e., the optimal channel/power/rate combination, by measuring the total noise-plus-interference level $\mathbf{M}_{i}$ over all channels. The best response of user $i$ is to maximize its individual utility function (4) subject to the constraints C1-C3. The same procedure is repeated for all users in the network. If such a procedure converges, then by definition, it has to converge to a $\mathrm{NE}$ of the game in (5).

Note that the utility function in (1) is monotonically increasing with $P_{i}\left(f_{k}\right)$ given that the other users' powers are 
fixed, and the only condition that prevents user $i$ from choosing infinitely large transmission power is the total power constraint C2. In our work, after adding the linear pricing function, the utility function (4) now leads to finite optimal power settings even without the constraint $\mathrm{C} 2$.

Proposition 3: Treating other users' transmissions as interference, the best response of user $i$ is given by:

$$
\mathbf{P}_{i}=\mathbf{B R}_{i}\left(\mathbf{P}_{-i}\right)=\left[B R_{i}\left(\mathbf{P}_{-i}\right)\left(f_{1}\right), \ldots, B R_{i}\left(\mathbf{P}_{-i}\right)\left(f_{K}\right)\right]
$$

with

$$
B R_{i}\left(\mathbf{P}_{-i}\right)\left(f_{k}\right)=\left[\frac{1}{\beta+\lambda_{i}\left(f_{k}\right)}-\frac{M_{i}\left(f_{k}\right)}{h_{i i}\left(f_{k}\right)}\right]_{0}^{P_{m a s k}\left(f_{k}\right)}
$$

where $[x]_{a}^{b}$, with $b>a$, denotes the Euclidean projection of $x$ onto the interval $[a, b]$, i.e., $[x]_{a}^{b}=a$ if $x<a,[x]_{a}^{b}=x$ if $a \leq x \leq b$, and $[x]_{a}^{b}=b$ if $x>b$. The water level $\beta$ is chosen to satisfy the total power constraint $\mathrm{C} 2$.

A similar result for the IWF algorithm is provided in [24]. Although we have an additional pricing function, a similar analysis can be used to reach the result in Proposition 3. We also provide an alternative proof in the Appendix using the sequential optimization technique as discussed in [7] and [27].

Note that without the power mask constraint and without the pricing function (i.e., $\lambda_{i}\left(f_{k}\right)=0$ for all $k$ and $i$ ), (13) becomes the classical water-filling solution. Figure 3 graphically illustrates the difference between the traditional water-filling [31] and the price-based water-filling solution (13). The variable water level in the right-hand side of Figure 3 is because of the addition of the pricing factor in (14).

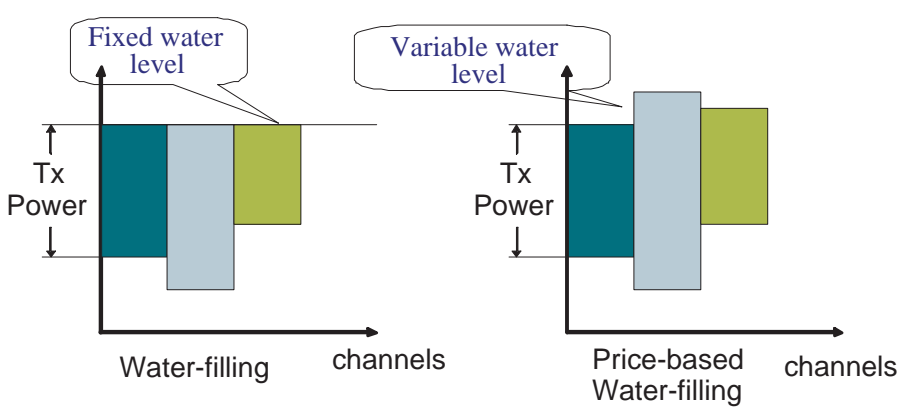

Fig. 3: IWF versus PIWF.

Several approaches are available for CR users to converge to the NE according to the best response function defined in (13). Naturally, CR users may make their decisions one after another or in parallel, which corresponds to sequential and parallel update procedures. The specific algorithms will be described next along with their convergence properties.

\section{A. Sequential Price-based Iterative Water-filling}

If $\mathrm{CR}$ users are to make their best-response decisions sequentially according to a fixed order, we have a sequential PIWF algorithm, and the algorithm can be generalized as follows:

The condition $\frac{\left\|\mathbf{P}_{i}^{(l)}-\mathbf{P}_{i}^{(l-1)}\right\|}{\left\|\mathbf{P}_{i}^{(l-1)}\right\|} \leq \varepsilon$ is the stopping criteria for the PIWF algorithm. Normally, $\varepsilon$ is set to a small value, such

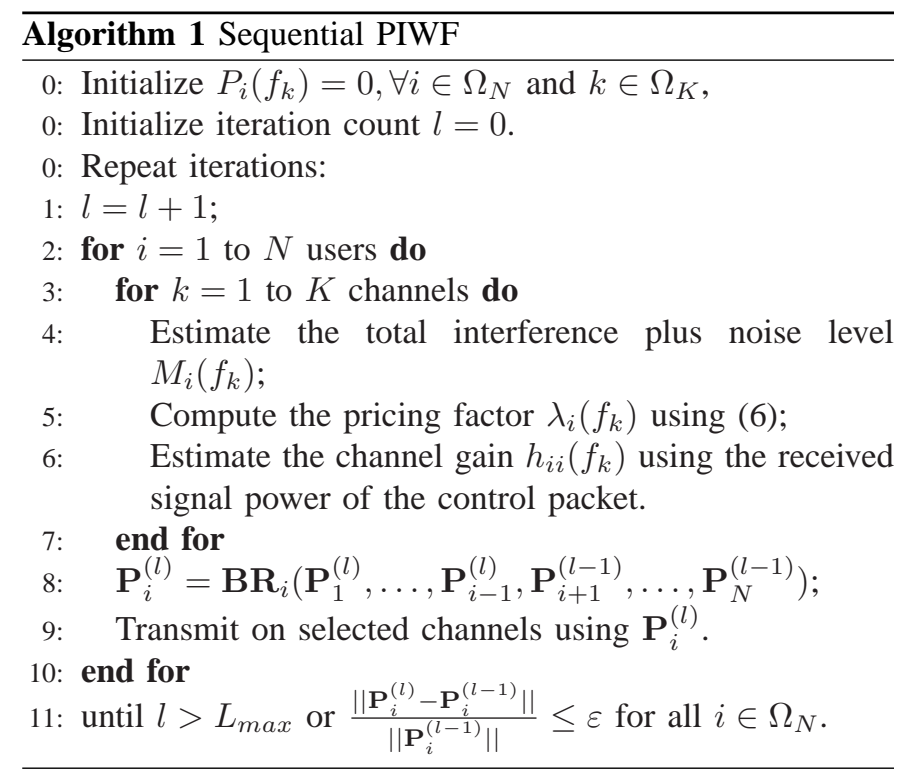

as $5 \%$. If that condition is not satisfied after $L_{\max }$ iterations, the algorithm terminates. The above algorithm is akin to the Gauss-Seidel procedure [13], where the players take their turns sequentially and act on the most recent policy information obtained from the other players. In a two-user scenario, the $(l+1)$ th iteration for user 1 can be expressed as:

$\mathbf{P}_{1}^{(l+1)}=\mathbf{B R}_{1}\left(\mathbf{B R}_{2}\left(\mathbf{P}_{1}^{(l)}\right)\right)=\left(\mathbf{B R}_{1} * \mathbf{B R}_{2}\right)\left(\mathbf{P}_{1}^{(l)}\right)=\mathbf{T}\left(\mathbf{P}_{1}^{(l)}\right)$

The Nash equilibrium is thus a fixed point [26] under the mapping $\mathbf{T}($.$) . For the N$-user case, the expression is more complicated, but we will keep the notation $\mathbf{T}$ as the mapping between the previous power vector and the current power vector. For the IWF algorithm, to ensure convergence to the NE, several sufficient conditions were proposed in the literature. The convergence condition was first provided in [31] for two-user cases and in [6] for $N$-user cases. More recently, the convergence conditions have been further relaxed in [12] and [24].

Since the utility function (4) in our formulation possesses an additional pricing function, the previous convergence proofs may not be applicable any more. In fact, since we are using a time-varying pricing factor in Algorithm 1, the mapping function $\mathbf{T}($.$) is also time-varying. Thus, the fixed point$ theorem that underlies the proofs in [12] and [24] can no longer be used. The convergence proof with a time-varying mapping function is challenging and will be left for a future work. However, convergence is always observed in our simulations for various network conditions. Figure 4 depicts the convergence behavior over several iterations with $N=10$ and $K=5$. In the test network, ten $\mathrm{CR}$ pairs are randomly placed in a square area. The figure shows the average sumrate improvement of the sequential PIWF over the classic IWF algorithm for 1000 runs, with the starting sum-rate of the IWF algorithm normalized to one. The two algorithms converge at comparable speeds, but the NE solution for the sequential PIWF algorithm is much better than the NE of the classic IWF algorithm. 


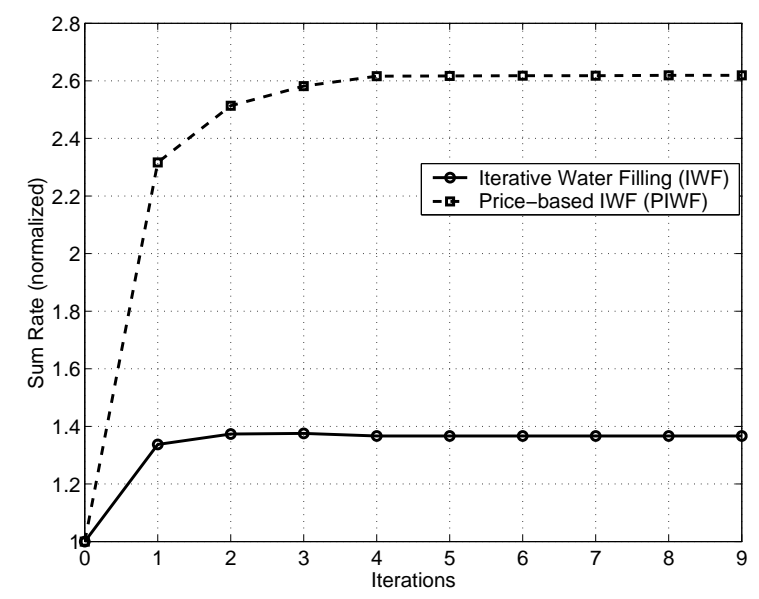

Fig. 4: Normalized system sum-rate versus iterations.

Although the convergence proof for the time-varying pricing function is difficult to establish, if the pricing factor remains fixed over a few iterations, the convergence proof in [24] is still applicable. This is because adding a linear pricing function with a fixed pricing factor to the utility function in (1) has no impact on the convergence proof in [24]. If we take the result in [24] and apply it to our CRN setting, we have the following proposition:

Proposition 4: Given a linear pricing function with a fixed pricing factor over a few iterations, the sequential update procedure converges to the unique $\mathrm{NE}$ if one of the two following sets of conditions is satisfied:

$$
\begin{gathered}
\sum_{j \in \Omega_{N}, j \neq i} \max _{k \in S_{i} \cap S_{j}} \frac{h_{j i}\left(f_{k}\right)}{h_{i i}\left(f_{k}\right)}<1, \forall i \in \Omega_{N} \\
\sum_{i \in \Omega_{N}, i \neq j} \max _{k \in S_{i} \cap S_{j}} \frac{h_{j i}\left(f_{k}\right)}{h_{i i}\left(f_{k}\right)}<1, \forall j \in \Omega_{N}
\end{gathered}
$$

From (16) and (17), the convergence and the uniqueness of the NE are ensured if the CRs that share the same channel are far apart from each other, and thus cause weak interference on one another. Figure 5 graphically illustrates these two conditions in a CRN that consists of three CR links. Each link is allocated two channels (e.g., nodes $A$ and $B$ are allocated channels 1 and 3). Condition (16) indicates that for each CR receiver, the summation of the normalized channel gains between that receiver and all interfering CR transmitters that share the same channel (normalized by the channel gain of the intended packet) is less than 1. For example, for node $B$, this condition reduces to $\frac{h_{C B}\left(f_{1}\right)}{h_{A B}\left(f_{1}\right)}+\frac{h_{E B}\left(f_{3}\right)}{h_{A B}\left(f_{3}\right)}<1$. Similarly, this condition is applied to receivers $D$ and $F$. If this condition is satisfied at all CR receivers, then according to Proposition 4 the sequential update procedure converges to the unique NE. The second condition (17) indicates that for each CR transmitter, the summation of the normalized channel gains between that transmitter and unintended CR receivers sharing the same channel is less than 1. For example, for transmitter $A$ (17) reduces to $\frac{h_{A D}\left(f_{1}\right)}{h_{C D}\left(f_{1}\right)}+\frac{h_{A F}\left(f_{3}\right)}{h_{E F}\left(f_{3}\right)}<1$. Similarly, we can derive this condition for transmitters $C$ and $E$. If this condition is satisfied at all CR transmitters, it is also sufficient to assert that the sequential update procedure converges to the unique NE.

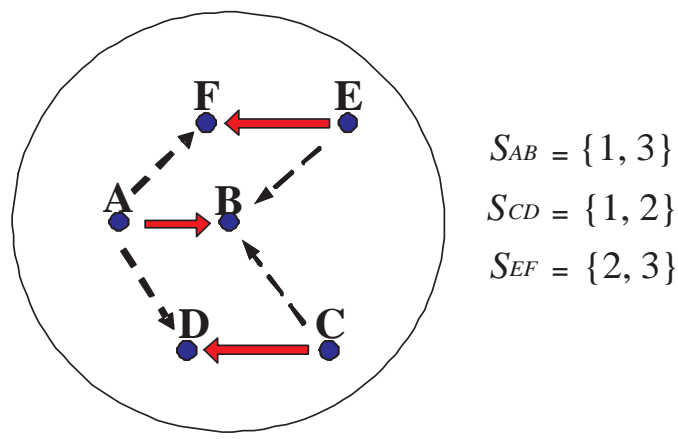

Fig. 5: Example network with three CR links.

If the number of users in the network is large, the sequential update procedure may suffer from slow convergence. Therefore, we now discuss a parallel version of the PIWF algorithm.

\section{B. Parallel Price-based Iterative Water-filling}

Algorithm 2 describes a parallel update version of the PIWF algorithm (the parallel IWF concept was first introduced in [25]). The stopping criteria for the parallel PIWF are the same as those of the sequential PIWF. The parallel PIWF algorithm is related to the Jacobi computational procedure [9], where in each iteration CR users simultaneously perform price-based water-filling based on the interference generated by the other users in the previous iterations. In a two-user case, the counterpart of (15) is:

$$
\mathbf{P}_{1}^{(l+2)}=\mathbf{B R}_{1}\left(\mathbf{P}_{2}^{(l+1)}\right)=\mathbf{B R}_{1}\left(\mathbf{B R}_{2}\left(\mathbf{P}_{1}^{(l)}\right)\right)=\mathbf{T}\left(\mathbf{P}_{1}^{(l)}\right)
$$

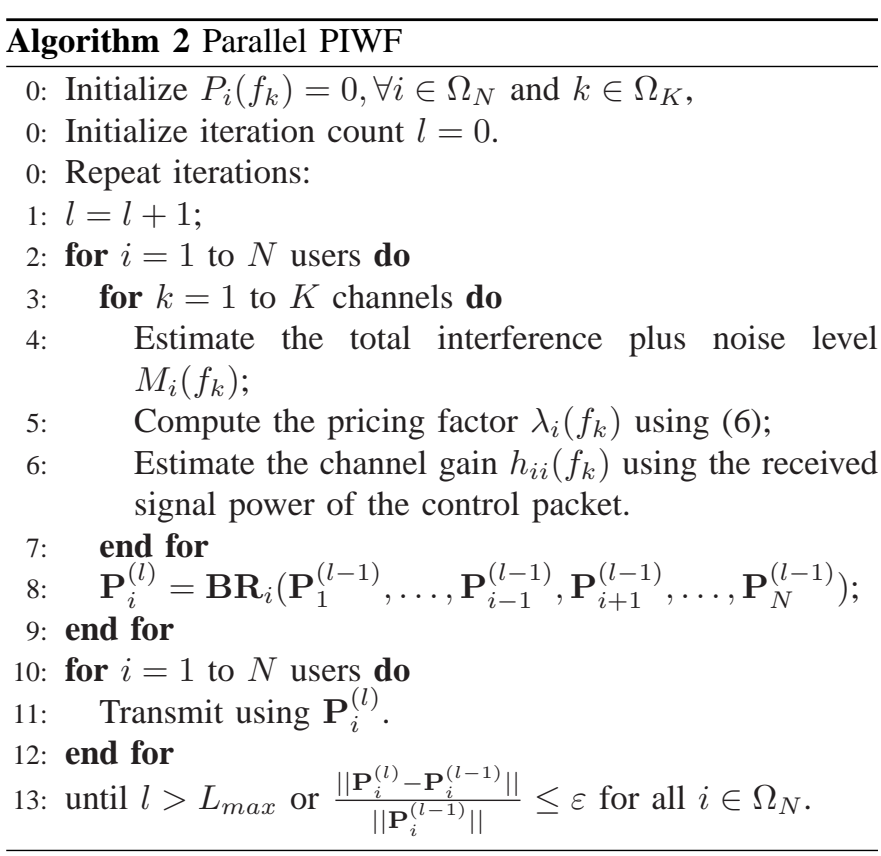

In [24], it was proved that the convergence conditions for the parallel IWF and the sequential IWF are the same. For 
a time-varying PIWF, the proof is not applicable. But if the pricing factor of the linear pricing function remains fixed over a few iterations, we can apply the corresponding proof and arrive at the following corollary of Proposition 4.

Corollary 1: If the conditions of Proposition 4 are satisfied, the parallel update procedure converges to the unique $\mathrm{NE}$ of the game.

Corollary 1 shows that stability under the Gauss-Seidel procedure coincides with stability under the Jacobi iteration. Furthermore, following the argument in [24], one can prove that any asynchronous computation in which the players act at random and use the most recent available policy from other players converges to a $\mathrm{NE}$, as long as no players remain idle for an infinite duration. Hence, the achieved NE based on asynchronous updates coincides with the NE achieved with parallel or sequential updates.

The parallel and sequential PIWF procedures are distributed algorithms that maximize the total achievable data rate. Both have the same implementation complexity of the traditional IWF. As shown in Figure 4, these two algorithms greatly outperform IWF. In Figure 6, we can see that the parallel PIWF converges faster than the sequential PIWF, especially for a large number of users. In this simulation, we assume that CRs are randomly located in a square area and 5 channels are available for their transmissions. Whether the players act sequentially or in parallel makes a difference in the MAC design. In Section $\mathrm{V}$, we discuss the impact of the update procedure on the MAC design.

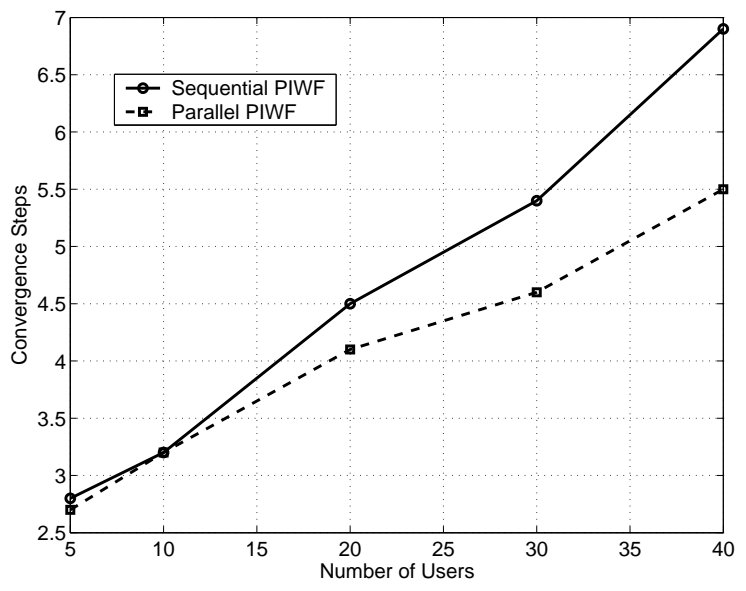

Fig. 6: Convergence speed of the sequential/parallel PIWF.

\section{Relaxation Algorithms}

Both the sequential and parallel PIWF algorithms require the system parameters to be correctly estimated for each CR. This condition may not be satisfied in practical systems. To overcome this problem, a "relaxed" update scheme can be used (as in [2], [15], and [24]), and will be discussed here for completeness. In such a "relaxed" version, each CR is required to remember its most recent policy choices together with the choices of other users. The relaxed algorithms are more robust to occasional estimation errors and channel oscillations, but lead to certain degradation in the convergence speed.
More specifically, we can achieve a relaxed version of the sequential PIWF algorithm if the best response function in Algorithm 1 is replaced by:

$$
\begin{aligned}
& \mathbf{P}_{i}^{(l)}=\omega \mathbf{P}_{i}^{(l-1)} \\
& +(1-\omega) \mathbf{B R}_{i}\left(\mathbf{P}_{1}^{(l)}, \ldots, \mathbf{P}_{i-1}^{(l)}, \mathbf{P}_{i+1}^{(l-1)}, \ldots, \mathbf{P}_{N}^{(l-1)}\right)
\end{aligned}
$$

where the factor $\omega \in[0,1)$ can be interpreted as the memory factor. The larger the value of $\omega$, the longer the memory of the algorithm. With a larger $\omega$, the algorithm is more robust to estimation errors at the cost of slower convergence.

Similarly, we can arrive at a relaxed version of the parallel PIWF algorithm if the best response in Algorithm 2 is replaced by:

$$
\begin{aligned}
& \quad \mathbf{P}_{i}^{(l)}=\omega \mathbf{P}_{i}^{(l-1)} \\
& +(1-\omega) \mathbf{B R}_{i}\left(\mathbf{P}_{1}^{(l-1)}, \ldots, \mathbf{P}_{i-1}^{(l-1)}, \mathbf{P}_{i+1}^{(l-1)}, \ldots, \mathbf{P}_{N}^{(l-1)}\right)(20)
\end{aligned}
$$

As proved in [24], the relaxation algorithms converge to the unique NE of the game for any $\omega \in[0,1)$ under the conditions in Proposition 4.

All the above proposed iterative algorithms are rate-adaptive (RA), where the data rates of users are maximized under transmission power constraints. Similarly, we can design "marginadaptive" (MA) algorithms where users attempt to minimize their transmission powers while satisfying a target data rate. Both RA and MA algorithms follow similar mechanisms. Due to space limitations, we do not discuss MA algorithms in this paper.

\section{MAC Protocol Design}

In this section, we describe a MAC protocol that allows CR users to operate efficiently in an opportunistic CRN. This protocol implements the distributed channel/power allocation strategies discussed in the previous sections. It should be noted that a number of multi-channel MAC protocols have been proposed in the context of CRNs (e.g., [14], [33], [32], and [28]). Most of them do not allow multiple CR transmissions within the same neighborhood to overlap in frequency channels, so there is no interference among CR users. Such a restriction simplifies the MAC design, but limits its spectrum efficiency. A natural extension (analogous to the improvement offered by the POWMAC protocol [17] over the classic CSMA/CA) is to allow CR users to overlap in spectrum, provided that their mutual interference does not lead to collisions. The IWF algorithm [31] and the no-regret algorithm [19] were proposed as two possible enabling techniques. However, the works in [31] and [19] provide only channel/power allocation algorithms and do not offer a practical MAC design.

In this section, we incorporate our price-based channel/power allocation algorithms into an operational MAC protocol. Since the IWF algorithm is a special case of the proposed PIWF algorithm, our MAC protocol can be simplified to accommodate the classic IWF algorithm, thus complementing the work in [31].

\section{A. Assumptions}

We consider a CRN setting with the following features: 
- A dedicated control channel or a coordinated control channel [32] is used to support a community of CR users. Control packets are transmitted over the control channel using a pre-assigned power value $P_{\text {cont }}$.

- Channel gains between any two terminals are symmetric.

- The channel gain is static for the duration of several control packets and a flow of data packets.

\section{B. Protocol Overview}

Our MAC protocol uses three types of control packets for the handshaking between a CR transmitter and a CR receiver: Request-to-Send (RTS), Clear-to-Send (CTS), and Decideto-Send (DTS). Unlike in the classic CSMA/CA scheme and other multi-channel MAC protocols for CRNs, these control packets are not used to exclusively reserve channels (i.e., prevent neighboring CRs from accessing the reserved channels), but rather to exchange some information within the neighborhood. Such information is used by terminals to determine their transmission parameters.

The control packets are exchanged within a certain duration, referred to as the contention window $(\mathrm{CW})$. A $\mathrm{CW}$ can be initiated asynchronously by any $\mathrm{CR}$ user that has packets to transmit and that is not aware of any active CWs in its neighborhood. Such a user is referred to as a master user. Other CR users that follow the schedule of an ongoing CW are called slave users. Note that the master/slave designation of a user is dynamic, i.e., it changes with traffic and mobility conditions. The objective of the $\mathrm{CW}$ is to allow several pairs of CR nodes to repeatedly negotiate their transmission channels and powers. As shown in Figure 7, the $\mathrm{CW}$ is divided into two parts. The first part, referred to as the access window (AW), is used by CR nodes to compete for admission to the $\mathrm{CW}$ and initialize their transmission policies. The second part, referred to as the training window (TW), is used by the CR nodes to repeatedly negotiate their channel/power policies (as explained later). Note that the AW can be considered as the first iteration of the training process. CR nodes that have been successfully admitted during the $\mathrm{CW}$ transmit a flow of data packets over one or multiple data channels (as determined during the $\mathrm{CW}$ ) within a data window (DW). The durations for the AW and DW are changed adaptively, similar to the singlechannel POWMAC protocol [17]. As for TW, its size (in slots) is dictated by the convergence speed of the iterative resource allocation algorithm. In general, an unnecessarily large value increases the overhead, but does not necessarily improve the throughput (as shown in Figure 4). On the other hand, a small value may give sub-optimal results. In Section VI, we study the performance of the MAC protocol under various TW sizes.

\section{Operation Details}

1) Access Window: When a CR node $A$ intends to communicate with another node $B$, it first needs to contend during the AW. If node $A$ is not aware of any ongoing AW in its neighborhood, it initiates a new AW (i.e., it becomes a master user). Otherwise, node $A$ contends during one of the slots of the ongoing AW. In either case, node $A$ first backs off by a

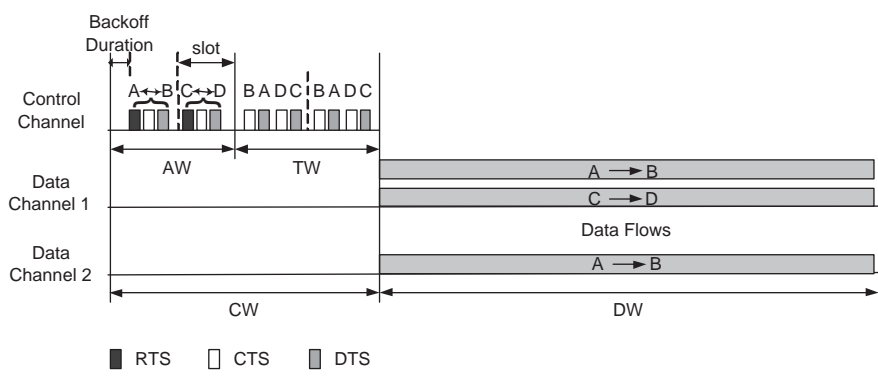

Fig. 7: Overview of the MAC operation with two CR transmissions $(A \rightarrow B$ and $C \rightarrow D)$.

random amount of time, selected from $\left[T_{\min }, T_{\max }\right]$, before accessing the channel.

The AW consists of a number of fixed-size slots. The size of each slot is $T_{\max }$ plus the durations of the RTS, CTS and DTS packets, plus 3 SIFS durations (SIFS denotes the short interframe spacing between successive control packets). In each slot, CR nodes compete for admission following a standard CSMA approach.

If CR $B$ successfully receives the RTS packet from $A$, it needs to decide the initial channel/power policy for the link $A \rightarrow B$. This is done as follows:

- First, node $B$ estimates the channel gain between itself and node $A$ (denoted by $h_{A B}\left(f_{0}\right)$ ). This is facilitated by knowledge of the RTS's transmission power $\left(P_{\text {cont }}\right)$ and the received power of the RTS. From $h_{A B}\left(f_{0}\right)$, CR $B$ computes $h_{A B}\left(f_{k}\right)$ for all $k \in \Omega_{K}$. The determination of $h_{A B}\left(f_{k}\right)$ from $h_{A B}\left(f_{0}\right)$ is made possible by knowing the carrier frequencies and by assuming a certain path-loss model. For example, under the two-ray model [21] and for a given transmission power, $h_{A B}\left(f_{k}\right)=h_{A B}\left(f_{0}\right) \times$ $\left(f_{0} / f_{k}\right)^{2}$, where $f_{0}$ is the carrier frequency of the control channel.

- Next, node $B$ measures $\mathbf{M}_{B}=$ $\left[M_{B}\left(f_{1}\right), M_{B}\left(f_{2}\right), \ldots, M_{B}\left(f_{K}\right)\right]$ over all data channels. Note that for the sequential PIWF algorithm, if there are previous CTS/DTS packets that have been received in the same $\mathrm{AW}, \mathbf{M}_{B}$ is computed as the sum of the current $\mathbf{M}_{B}$ and the predicted CR-to-CR interference, which is obtained by assuming that the neighboring links transmit using the channels/powers specified in their CTS/DTS.

- Then, node $B$ determines the pricing factor $\lambda_{B}\left(f_{k}\right)$ for all data channels $k$. For the sequential PIWF algorithm, $\lambda_{B}\left(f_{k}\right)$ is computed using (6), where the neighborhood information is obtained from previously received CTS/DTS packets in the same AW. For the parallel PIWF algorithm, $\lambda_{B}\left(f_{k}\right)$ is initialized to 0 .

- Finally, based on the above information, node $B$ decides its best-response transmission policy according to Proposition 3.

After the above procedures have been executed, node $B$ will send a CTS, announcing its channel/power allocation. The CTS includes $M_{B}\left(f_{k}\right)$ and $h_{A B}\left(f_{k}\right)$ for all $k \in S_{B}$, which are used by neighboring CRs to update their best responses. Note that even if the set of selected channels $S_{B}$ is empty (i.e., 
the computed transmission power is zero for all channels), the link $A \rightarrow B$ will still be admitted in the AW. This is because the data transmission $A \rightarrow B$ may later be allowed to proceed after several iterations in the TW.

If node $A$ receives the CTS from $B$, it will respond with a DTS packet, repeating the information included in the CTS. This DTS is used to alleviate the hidden terminal problem as in [17].

The above steps are repeated by CR pairs in every AW slot.

2) Training Window: CR nodes that are admitted in the AW iteratively negotiate their transmission parameters in the TW, following the same order of their admissions in the AW. In contrast to the AW, the TW is accessed in a TDMA manner. The TW consists of a number of slots (the TW size), where each slot is used to conduct one iteration of the channel/power allocation algorithm, using CTS and DTS packets. Note that there is no need for the RTS during the TW, since new admissions are not allowed.

In each iteration, the receiver of a CR link updates the transmission policies based on the policies of its neighbors. The updates are made based on either the sequential or the parallel scheme. Specifically, if the sequential PIWF algorithm is applied, the transmission policy of each CR user is made based on the policies of all previous users in the same iteration (obtained from CTS/DTS packets) and those of the other users in the previous iteration, as described in Algorithm 1. If the parallel PIWF algorithm is applied, the policy of each CR user is made based on the policies of other CR users in the previous iteration, as described in Algorithm 2. Note that the AW is regarded as the initial iteration of the training process. After each computation, the receiver sends a CTS, announcing its transmission policy. Upon receiving the CTS, the transmitter will send a DTS, repeating the information in the CTS.

3) Data Window: The last negotiated transmission policies in the TW are used by the CR nodes for data transmissions in the DW. In the DW, a flow of data packets is transmitted from each CR transmitter. The length of the flow is selected such that the channel conditions remain static over the entire flow. Obviously, the DW size needs to be selected according to the channel's coherence time.

\section{Simplified Packet-based MAC Design}

The above MAC design can be used for flow-based channel access, where a sequence of data packets are transmitted using converged channel/power policies agreed upon during the TW. Thus, the sum-rate of all competing CRs is likely to be maximized if the channels remain static over the duration of the data flow. However, if sum-rate optimality is not critical, we can simplify the protocol by removing the TW and only allow for a single data-packet transmission in the DW. This design then becomes packet-based, and the convergence is now achieved after several sessions of CW and DW (provided that channel conditions remain static within this period).

Note that in the previous section, all CR nodes contend in the AW with equal probability. In contrast, in the packetbased MAC design, the admitted users in the previous AW have priorities in accessing the control channel over other
CR users. Specifically, the admitted links in the previous AW will contend in the current AW without backoff, according to their order in the previous AW, as long as they still have packets to transmit. After these links have been admitted, other links compete for the remaining slots, following the backoff mechanism that was discussed in the previous section. Such a design is meant to facilitate the convergence behavior. To ensure fairness among users, we set a limit $(\theta)$ on the maximum number of continuously prioritized packets. Specifically, if one CR user acquires the channel in one session, it will have priorities in accessing the control channel for the next $\theta-1$ packet transmission durations (as long as the user has packets to transmit). The parameter $\theta$ is selected to be larger than the convergence time of the algorithm.

The channel/power policies are updated in the AW following similar procedures to the flow-based MAC. The only difference is that the interference-plus-noise level is now estimated from the previous DW, instead of the previous iteration in the TW. In the next section, we compare the performance of this design with that of the flow-based MAC.

\section{E. Implementation of Relaxed Algorithms}

The implementation of the "relaxed" version algorithms to the MAC design is straightforward. Each CR receiver memorizes its most recent policy, and makes decisions based on (19) for the sequential algorithm (or (20) for the parallel algorithm). Since the convergence speed of the "relaxed" PIWF is slower, a larger TW size is needed for the flow-based MAC protocol. Similarly, for the packet-based MAC, a larger number of data packets are transmitted before the convergence can be achieved.

\section{PERFormance EVAluation}

To evaluate the effectiveness of the proposed MAC, we conduct MATLAB-based simulations of a hybrid network with one PRN and one CRN. Nodes in these networks are uniformly distributed over a square area of length 100 meters. The PRN operates in the $300 \mathrm{MHz}$ band, occupying five non-overlapping $1-\mathrm{MHz}$ channels, with $10 \mathrm{PRs}$ in each channel. The time is divided into slots, each of length $10 \mathrm{~ms}$. In each slot, each PR attempts to transmit with a probability of $\alpha$, the PR's activity factor. The transmission power of each PR is 1 Watt when it is on, and the antenna length is $5 \mathrm{~cm}$.

We use the following signal propagation model to simulate the PR-to-CR and CR-to-CR interference over channel $k$ [21]:

$$
P_{r}\left(f_{k}\right)=P_{d_{0}}\left(f_{k}\right)\left(\frac{d}{d_{0}\left(f_{k}\right)}\right)^{-\gamma}, \text { for } d>d_{0}\left(f_{k}\right)
$$

where $P_{r}\left(f_{k}\right)$ is the received power over channel $k, d$ is the distance between the transmitter and the receiver, $d_{0}\left(f_{k}\right)$ is the reference distance, $P_{d_{0}}\left(f_{k}\right)$ is the reference received power at distance $d_{0}\left(f_{k}\right)$ over channel $k$, and $\gamma$ is the path loss exponent. We set $d_{0}\left(f_{k}\right)=1$ meter, $\gamma=4$, and we compute $P_{d_{0}}\left(f_{k}\right)$ as follows [21]:

$$
P_{d_{0}}\left(f_{k}\right)=P_{t}\left(f_{k}\right) G_{t}\left(f_{k}\right) G_{r}\left(f_{k}\right)\left(\frac{\nu\left(f_{k}\right)}{4 \pi d_{0}\left(f_{k}\right)}\right)^{2}
$$


where $P_{t}\left(f_{k}\right)$ is the transmission power on channel $k, G_{t}\left(f_{k}\right)$ and $G_{r}\left(f_{k}\right)$ are the transmitter and receiver antenna gains on channel $k$, and $\nu\left(f_{k}\right)$ is the wavelength of the carrier frequency of channel $k$. For simplicity, we set $G_{t}\left(f_{k}\right) G_{r}\left(f_{k}\right)=1$ for all channels.

We simulate 10 pairs of CRs. The maximum transmission power for $\mathrm{CR}$ is 1 Watt, which ensures that CRs are within the maximum transmission range of each other. The AWGN noise level $N_{0}$ is set to $-70 \mathrm{dBm}$ over all channels. Each CR transmitter generates burst of packets according to a Poisson process with parameter $\Lambda$ burst/second. Each burst has an exponentially distributed duration with mean $1 / \mu$ second. The traffic rate for CR is defined as $\Lambda / \mu$. We set the CR-to-PR power mask to 0.5 Watt for all channels.

We compare the performance of the proposed flow-based PIWF-MAC protocol with the packet-based PIWF-MAC protocol, against the flow-based IWF-MAC protocol. Since the IWF algorithm is a special case of the PIWF algorithm, the proposed MAC protocols are also applicable to the IWF algorithm. The DW for the flow-based MAC protocol allows 10 data packets to be transmitted in a row. The comparison is in terms of the system throughput and the average power consumption. The system throughput is defined as the average volume of CR traffic bits that are transmitted in one second, and the power consumption is calculated as average power consumption by all CRs.

The resulting performance is depicted in Figure 8 through Figure 10. Figure 8(a) shows the system throughput versus the traffic rate. As expected, the flow-based PIWF-MAC protocol gives the highest throughput. This throughput improvement over IWF-MAC becomes more significant at higher traffic rates. It is interesting to see that the simplified packet-based PIWF-MAC protocol exhibits comparable system throughput with the flow-based PIWF-MAC protocol. Besides achieving a higher system throughput, the PIWF-MAC protocols also save transmission power, as shown in Figure 8(b). This is because in IWF, users greedily maximize their own rates using the maximum transmission power, while such greedy behaviors are overcome by the pricing technique used in PIWF. Note that although the packet-based PIWF-MAC consumes less energy in control overhead than the flow-based PIWF-MAC, it consumes more energy in data transmissions. This is because in the packet-based PIWF-MAC, the optimal power assignment is achieved after several packet transmissions (due to the absence of a control-based training phase). The confluence of the two energy-consumption factors still favors the flow-based PIWFMAC.

Figure 9(a) depicts the network throughput versus the PR's activity factor $\alpha$. As expected, a higher $\alpha$ results in a higher PR-to-CR interference, which negatively affects the throughput. Figure 9(b) shows the corresponding average power consumption. In all cases, PIWF-MAC protocols consume less power.

Finally, Figure 10(a) shows the throughput versus the training window size. Since the simplified packet-based PIWFMAC does not employ a TW, we only compare the flowbased PIWF-MAC and flow-based IWF-MAC. Intuitively, a larger TW size will ensure that CR users will converge to the
NE. However, as seen in Figure 4, 2-3 iterations are normally sufficient to reach a near-optimal sum-rate. The same behavior is observed from the MAC simulations. As seen in Figure 10(a), taking a TW of size 2 is enough to achieve $95 \%$ of the maximum system throughput in the simulation setup. Figure 10(b) shows the corresponding average power consumption.

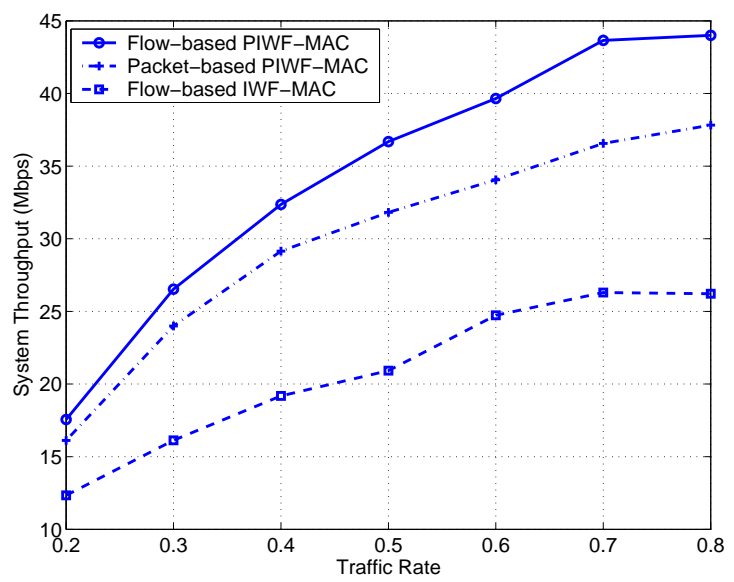

(a) System throughput vs. traffic rate.

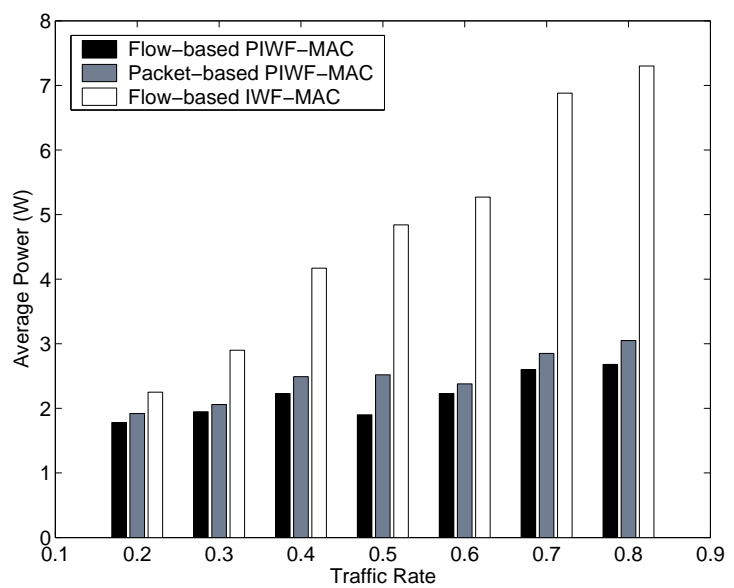

(b) Average power consumption vs. traffic rate.

Fig. 8: Performance when $\alpha=0.1$.

\section{CONCLUSIONS}

In this paper, we proposed a PIWF algorithm for spectrum sharing in cognitive radio networks. Our PIWF algorithm can be implemented distributively with CRs repeatedly negotiating their transmission powers and spectrum. Simulation results showed that the proposed algorithm greatly improves the NE compared with the one achieved using the IWF approach. Based on the order by which CR nodes make their resource allocation decisions, we studied sequential and parallel versions of the PIWF algorithm. The parallel update scheme was shown to converge faster than the sequential update scheme, especially for a large number of users. We also presented "relaxed" versions of the PIWF algorithms, which are more robust to estimation errors and channel oscillations at the cost of slower convergence. Based on the PIWF algorithms, flow-based and packet-based MAC protocols were designed. 


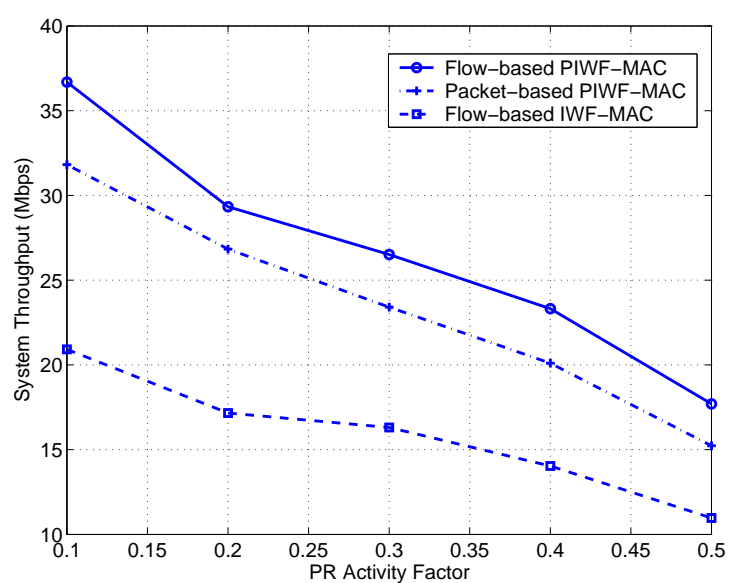

(a) System throughput vs. PR activity factor.

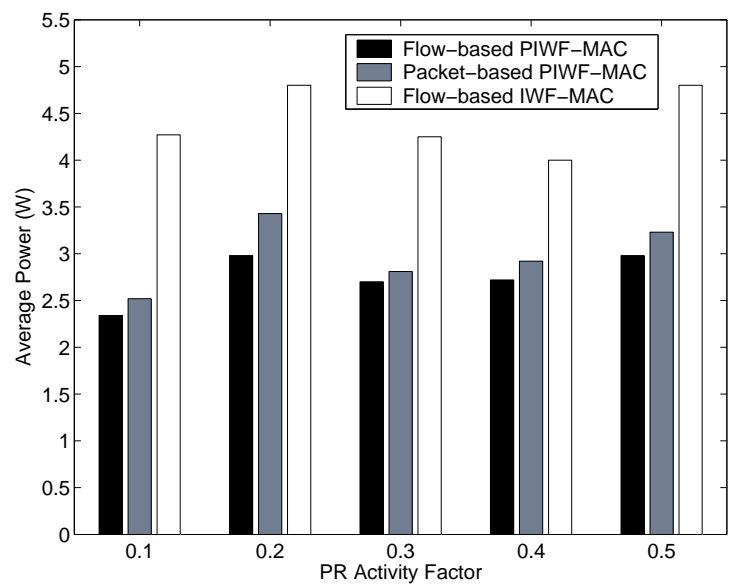

(b) Average power consumption vs. PR activity factor.

Fig. 9: Performance under traffic rate $\Lambda / \mu=0.5$.

Our simulation results showed that the PIWF-MAC protocol achieves considerably higher system throughput compared with the IWF-MAC, with less energy consumption.

\section{APPENDIX}

\section{A. Proof of Proposition 3}

Proof: We first solve the optimization problem without the power mask constraint $\mathrm{C} 3$, using the method of Lagrange multipliers. This leads to a water-filling solution of the form [31]:

$$
P_{i}^{*}\left(f_{k}\right)=\left[\frac{1}{\beta+\lambda_{i}\left(f_{k}\right)}-\frac{M_{i}\left(f_{k}\right)}{h_{i i}\left(f_{k}\right)}\right]^{+} .
$$

If $P_{i}^{*}\left(f_{k}\right)$ is the optimal power allocation over channel $k$, then the slope of the utility function $u_{i}\left(P_{i}\left(f_{k}\right)\right)$ must be positive at the point $P_{i}^{*}\left(f_{k}\right)$. Otherwise, a power vector $\mathbf{P}_{i}$ with a smaller $P_{i}\left(f_{k}\right)$ could reach a higher utility $U_{i}\left(P_{i}\right)$, with all the constraints satisfied. Thus, the utility function $u_{i}\left(P_{i}\left(f_{k}\right)\right)$ is monotonically increasing between 0 and $P_{i}^{*}\left(f_{k}\right)$. Accordingly, if any of the $P_{i}^{*}\left(f_{k}\right)$ in (23) violates the upper bound C3, then the corresponding bounded optimal solution must be the upper bound $P_{\text {mask }}\left(f_{k}\right)$ itself (a similar approach was also adopted

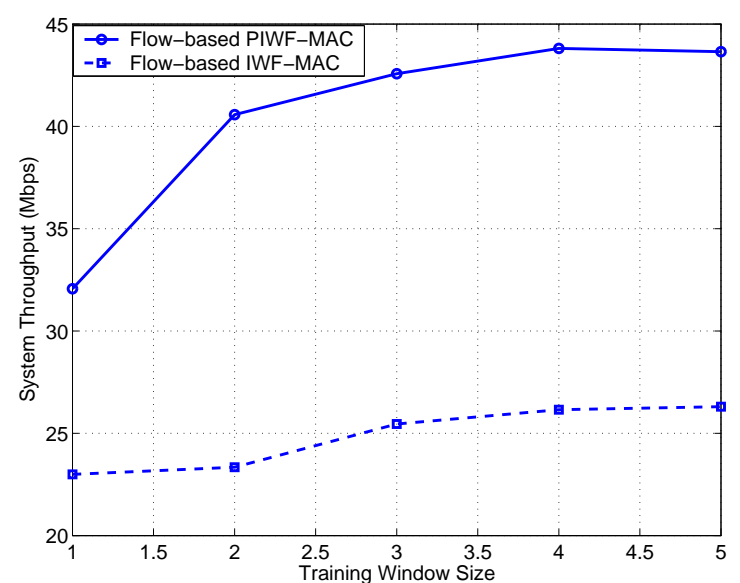

(a) System throughput vs. TW size.

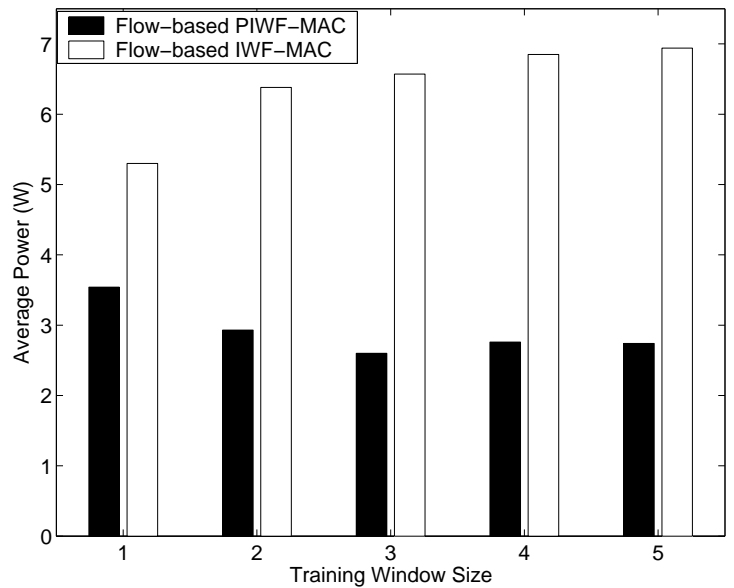

(b) Average power consumption vs. TW size.

Fig. 10: Performance under traffic rate $\Lambda / \mu=0.7$ and $\alpha=$ 0.1 .

in [7]). After bounding $P_{i}^{*}\left(f_{k}\right)$ by $P_{\text {mask }}\left(f_{k}\right)$, the remaining power will be further water-filled over other channels, thus reaching the result in (13).

\section{REFERENCES}

[1] P. Antoniadis, C. Courcoubetis, and R. Mason. Comparing economic incentives in peer-to-peer networks. Computer Networks, 46(1):133146, 2004

[2] T. Basar. Relaxation techniques and asynchronous algorithms for online computation of non-cooperative equilibria. Journal of Economic Dynamics and Control, 11(4):531-549, 1987.

[3] S. Boyd and L. Vandenberghe. Convex Optimization. Cambridge University Press, 2004.

[4] R. Cendrillon, W. Yu, M. Moonen, J. Verlinden, and T. Bostoen. Optimal multi-user spectrum management for digital subscriber lines. IEEE Transactions on Communications, 50(2):291-303, February 2006.

[5] Q. Chen and Z. Niu. A game-theoretical power and rate control for wireless ad hoc networks with step-up price. IEICE Transactions on Communications, E88-B(9):3515-3523, September 2005.

[6] S. T. Chung, S. J. Kim, J. Lee, and J. M. Cioffi. A game-theoretic approach to power allocation in frequency-selective Gaussian interference channels. In Proceedings of the IEEE International Symposium on Information Theory (ISIT 2003), page 316, June 2003.

[7] S. Cui, J. Xiao, A. J. Goldsmith, Z.-Q. Luo, and H. V. Poor. Estimation diversity and energy efficiency in distributed sensing. IEEE Transactions on Signal Processing, 55(9):4683 - 4695, September 2007.

[8] FCC. ET Docket No. 03-322, Notice of proposed rule making and order December 2003. 
[9] L. Hageman and D. Young. Applied Iterative Methods. Academic Press, New York, 1981.

[10] S. Haykin. Cognitive radio: Brain-empowered wireless communications. IEEE Journal on Selected Areas in Communications, 23(2):201-220, February 2005.

[11] J. Hirshleifer, A. Glazer, and D. Hirshleifer. Price Theory and Applications Decisions, Markets, and Information. Cambridge University Press, November 2005.

[12] J. Huang, R. Cendrillon, M. Chiang, and M. Moonen. Autonomous spectrum balancing (ASB) for frequency selective interference channels. In Proceedings of the IEEE International Symposium on Information Theory (ISIT 2006), July 2006.

[13] W. Kahan. Gauss-Seidel Methods of Solving Large Systems of Linear Equations. Ph.D. Dissertation, University of Toronto, Toronto, Canada, 1958.

[14] S. Krishnamurthy, M. Thoppian, S. Venkatesan, and R. Prakash. Control channel based MAC-layer configuration, routing and situation awareness for cognitive radio networks. In Proceedings of the Military Communications Conference (MILCOM), volume 1, pages 455-460, Oct 2005.

[15] R. Maheswaran and T. Basar. Decentralized network resource allocation as a repeated noncooperative market game. In Proceedings of the 40th IEEE Conference on Decision and Control, volume 5, pages 4565-4570, Orlando, FL, Dec 2001

[16] J. Mitola. Cognitive radio: An integrated agent architecture for software defined radio. Ph.D. Dissertation, KTH Royal Institute of Technology, Stockholm, Sweden, 2000.

[17] A. Muqattash and M. Krunz. POWMAC: A single-channel powercontrol protocol for throughput enhancement in wireless ad hoc networks. IEEE Journal on Selected Areas in Communications, 23(5):10671084, May 2005.

[18] J. Neel, J. Reed, and R. Gills. Convergence of cognitive radio networks. In Proceedings of the Wireless Communications and Networking Conference, Atlanta, GA, March 2004.

[19] N. Nie and C. Comaniciu. Adaptive channel allocation spectrum etiquette for cognitive radio networks. In IEEE Symposium on New Frontiers in Dynamic Spectrum Access Networks (IEEE DySPAN), November 2005.

[20] M. J. Osborne. An Introduction to Game Theory. Oxford University Press, 2004.

[21] T. S. Rappaport. Wireless Communications - Principles and Practice. Prentice Hall, 2nd edition, 2001.

[22] J. B. Rosen. Existence and uniqueness of equilibrium points for concave N-person games. Econometrica, 33(3):520-534, July 1965.

[23] C. U. Saraydar, N. B. Mandayam, and D. J. Goodman. Efficient power control via pricing in wireless data networks. IEEE Transactions on Communications, 50(2):291-303, February 2002.

[24] G. Scutari, D. P. Palomar, and S. Barbarossa. Asynchronous iterative waterfilling for Gaussian frequency-selective interference channels: A unified framework. Submitted to IEEE Transactions on Information Theory, August 2006.

[25] G. Scutari, D. P. Palomar, and S. Barbarossa. Simultaneous iterative water-filling for Gaussian frequency-selective interference channels. In Proceedings of the IEEE International Symposium on Information Theory (ISIT 2006), pages 600-604, July 2006.

[26] Y. A. Shashkin. Fixed Points. Providence, November 1991.

[27] Y. Shen, P. C. Cosman, and L. B. Milstein. Error resilient video communications over CDMA networks with a bandwidth constraint. IEEE Transaction on Image Processing, 15(11):3241-3252, November 2006.

[28] T. Shu, S. Cui, and M. Krunz. Medium access control for multi-channel parallel transmission in cognitive radio networks. In Proceedings of the IEEE GLOBECOM Conference, November 2006.

[29] K. B. Song, S. T. Chung, G. Ginis, and J. M. Cioffi. Dynamic spectrum management for next generation DSL systems. IEEE Communication Magazine, 40(10):101-109, October 2002.

[30] F. Wang, O. Younis, and M. Krunz. GMAC: A game-theoretic MAC protocol for mobile ad hoc networks. In Proceedings of the 4th International Symposium on Modeling and Optimization in Mobile, Ad Hoc, and Wireless Networks (WiOpt06), April 2006.

[31] W. Yu. Competition and cooperation in multi-user communication environments. Ph.D. Dissertation, Stanford University, Stanford, CA, 2002.

[32] J. Zhao, H. Zheng, and G. Yang. Distributed coordination in dynamic spectrum allocation networks. In IEEE Symposium on New Frontiers in Dynamic Spectrum Access Networks (IEEE DySPAN), November 2005.
[33] Q. Zhao, L. Tong, A. Swami, and Y. Chen. Decentralized cognitive MAC for opportunistic spectrum access in ad hoc networks: A POMDP framework. IEEE Journal on Selected Areas in Communications, 25(3):589-600, April 2007.

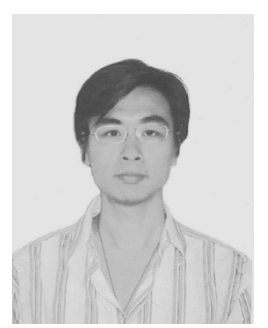

Fan Wang received the B.S. degree from Tsinghua University, China, in 2001 and the M.S. degree from the University of Arizona, Tucson, in 2004. He is currently working towards the Ph.D. degree in the Department of Electrical and Computer Engineering, University of Arizona.

His current research interests are in system architecture and communication protocol designs for cellular, ad hoc, and cognitive radio wireless networks with emphasis on spectrum, power and rate control. He has several journal articles and refereed conference papers in this area. One of his papers won the best student paper award in Second International Conference on Cognitive Radio Oriented Wireless Networks and Communications (CrownCom 2007). During summer of 2006, he was a member of the WiMAX development group, FREESCALE, Inc. F. Wang serves as a reviewer for many IEEE conferences and journals.

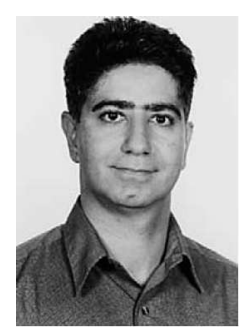

Marwan Krunz is a professor of electrical and computer engineering at the University of Arizona and the director of the advanced networking and wireless communications group. He received his $\mathrm{Ph} . \mathrm{D}$. degree in electrical engineering from Michigan State University in 1995. He joined the University of Arizona in January of 1997, following a brief postdoctoral stint at the University of Maryland, College Park. He previously held visiting research positions at INRIA (Sophia Antipolis, France), HP Labs (Palo Alto, California), Paris VI, and US West (now Qwest) Advanced Technologies. His research interests lie in the fields of computer networking and wireless communications. His recent interests include power/rate control in wireless and sensor networks, channel access and routing protocols, media streaming, quality of service routing, and optical networking. He previously worked on traffic analysis, modeling and performance evaluation, packet video modeling, and QoS provisioning in highspeed networks. M. Krunz is a recipient of the National Science Foundation CAREER Award (1998-2002). He currently serves on the editorial board for the IEEE/ACM Transactions on Networking, the IEEE Transactions on Mobile Computing, and the Computer Communications Journal. He was a guest coeditor for special issues in IEEE Micro and IEEE Communications Magazines. He served as a technical program chair for the IEEE WoWMoM 2006, the IEEE International Conference on Sensor and Ad hoc Communications and Networks (SECON 2005); the IEEE INFOCOM 2004 Conference; and the 9th Hot Interconnects Symposium, August 2001. He has served and continues to serve on the executive and technical program committees of many international conferences and on the panels of several NSF directorates. He gave several tutorials at premier wireless networking conferences. 


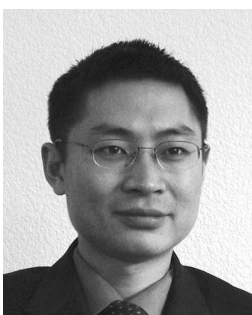

Shuguang Cui (S'99-M'05) received Ph.D in Electrical Engineering from Stanford University, California, USA, M.Eng in Electrical Engineering from McMaster University, Hamilton, Canada, in 2000, and B.Eng. in Radio Engineering with the highest distinction from Beijing University of Posts and Telecommunications, Beijing, China, in 1997. He is now working as an assistant professor in Electrical and Computer Engineering at the Texas A\&M University, College Station, TX.

From 1997 to 1998 he worked at Hewlett-Packard, Beijing, P. R. China, as a system engineer. In the summer of 2003, he worked at National Semiconductor, Santa Clara, CA, on the ZigBee project. From 2005 to 2007, he worked as an assistant professor at the department of Electrical and Computer Engineering, University of Arizona, Tucson, AZ. His current research interests include cross-layer energy minimization for lowpower sensor networks, hardware and system synergies for high-performance wireless radios, statistical signal processing, and general communication theories. He was a recipient of the NSERC graduate fellowship from the National Science and Engineering Research Council of Canada and the Canadian Wireless Telecommunications Association (CWTA) graduate scholarship. $\mathrm{He}$ has been serving as the TPC co-chairs for the 2007 IEEE Communication Theory Workshop and the ICC'08 Communication Theory Symposium. He is currently serving as the associate editors for the IEEE Communication Letters and IEEE Transactions on Vehicular Technology. 